\title{
COVID-19 outcomes in patients with hematologic disease
}

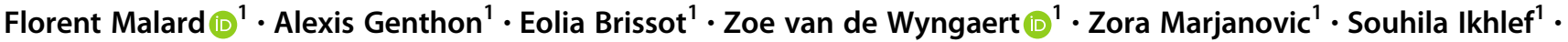 \\ Anne Banet ${ }^{1}$ - Simona Lapusan ${ }^{1}$ - Simona Sestilli ${ }^{1}$ - Elise Corre ${ }^{1}$. Annalisa Paviglianiti ${ }^{1} \cdot$ Rosa Adaeva $^{1}$. \\ Fella M.'Hammedi-Bouzina ${ }^{1} \cdot$ Myriam Labopin $^{1} \cdot$ Ollivier Legrand $^{1} \cdot$ Rémy Dulery $^{1} \cdot$ Mohamad Mohty $^{1}$
}

Received: 16 April 2020 / Revised: 23 April 2020 / Accepted: 27 April 2020 / Published online: 6 May 2020

(c) Springer Nature Limited 2020

\section{To the Editor:}

Severe acute respiratory syndrome coronavirus-2 (SARS$\mathrm{CoV}-2)$ is a novel coronavirus of zoonotic origin that emerged in China at the end of 2019. The infection, named Coronavirus Disease 2019 (COVID-19), is now spreading worldwide. As of April 16, 2020, the virus had affected more than 2,000,000 individuals and resulted in over 125,000 deaths worldwide. Mortality can be as high as $15 \%$ in elderly patients, and/or in patients with comorbidities $[1,2]$. Based on the current available data, the incubation period (time from exposure to symptom development) is estimated as between 2 and 14 days [3]. At present, there are no approved treatment options in Europe and no available vaccine. Avoiding exposure by adhering to recommended hygiene procedures, isolation of infected persons and social distancing are the only prevention strategies recommended by the WHO [4].

Risk factors for COVID-19 severity and death include older age, along with comorbidities such as diabetes, hypertension, or cardiac disease $[1,2]$. In addition, data from China suggest that patients with cancer have a significantly higher incidence of severe events (including intensive care unit admission, need of assisted ventilation,

These authors contributed equally: Florent Malard, Alexis Genthon

These authors jointly supervised this work: Rémy Dulery, Mohamad Mohty

Supplementary information The online version of this article (https:// doi.org/10.1038/s41409-020-0931-4) contains supplementary material, which is available to authorized users.

$\triangle$ Florent Malard

florent.malard@inserm.fr

1 Service d'Hématologie Clinique et Thérapie Cellulaire, Hôpital Saint-Antoine, Sorbonne Université, INSERM UMRs 938, Paris, France death) after contracting the virus (39\% versus $8 \%$ in patients without cancer) [5]. Another study reported that cancer patients appear to be twice as likely to contract infection with SARS-CoV-2 [6]. Importantly, that study suggests that hospital admission and recurrent hospital visits, inherent to cancer patients' management, are potential risk factors for SARS-CoV-2 infection [6]. To date, very few data are available on COVID-19 outcomes in patients with hematologic diseases. Only one 47-year-old patient with a lymphoma has been included in a previous report [5], and two articles have reported on the course of COVID-19 infection in a 39-year-old patient with chronic lymphocytic leukemia [7] and in a 60-year-old patient with multiple myeloma (MM) [8]. All three patients had a favorable outcome. Nevertheless, these were relatively young, unlike the overall patient population with hematologic neoplasms which is usually aged, comorbid and highly immunosuppressed. These patients are therefore expected to be a particularly vulnerable group for COVID-19. A better characterization of those infected with the virus is important. Here we describe the demographic characteristics, coexisting conditions, imaging findings, and outcomes among patients with hematologic disease and COVID-19 infection.

We included all consecutive adult patients with a hematologic disease admitted to the Hematology Department (inpatient and outpatient admissions) of the SaintAntoine-Hospital, AP-HP, Paris, France, with laboratoryconfirmed COVID-19 infection between March 9 and April 4, 2020 and with at least 10 days of follow-up. A confirmed case of COVID-19 was defined by a positive result on a real-time RT-PCR assay of a specimen collected on a nasopharyngeal swab. We reviewed medical records to collect demographic, clinical, and treatment data and outcomes of COVID-19. All laboratory tests and radiologic assessments, including plain chest radiography and computerized chest tomography, were performed at the discretion of the treating physician. 
COVID-19 was suspected and screened by PCR in 48 patients with a hematologic disease and the infection was identified in 25. Clinical details on hematologic and treatment history and COVID-19 infection are listed in Table 1. The median patient age was 72 (range, 40-96) years, 68\% were male. The median duration of symptoms before the COVID-19 PCR assay was performed, was 4 (range, 0-22) days. None of the patients had recently traveled to a country with known transmission such as China, Iran, or Italy, but five had direct contact with a COVID-19 positive family member. Among the remaining patients, six were already hospitalized (none of them in the hematology department) at the time of viral infection symptoms' onset due to a fall episode $(n=2)$, MM diagnosis $(n=3)$ or accidental cardiac drug overdose $(n=1)$. Ten patients had one or more outpatient visits to the hematology department, suggesting a possible nosocomial origin of their infection. In the remaining four patients, the origin of COVID-19 infection was unknown.

The most common symptoms at diagnosis were fever $(n=22,89 \%)$, cough $(n=19,79 \%)$, and shortness of breath $(n=19,79 \%)$. The majority $(n=20,80 \%)$ of patients had a lymphoid malignancy, including 10 with MM $(40 \%)$, and only $4(16 \%)$ had a myeloid malignancy (myelodysplastic syndrome). One patient had paroxysmal nocturnal hemoglobinuria. Patients received a median of 1 (range, 0-6) line of treatment. Fourteen patients (56\%) were being treated for their underlying disease at the time of COVID-19 diagnosis, with $10(40 \%)$ receiving corticosteroids. Seven patients had a history of hematopoietic stem cell transplantation (autologous, $n=5$, allogeneic, $n=1$, and both, $n=1$ ) and one had been treated with anti-CD19 CAR $\mathrm{T}$ cells 3 months before. Of note, the four patients with myelodysplastic syndrome received only supportive care, one patient with MM had just been diagnosed and had not initiated therapy, and one with stage A chronic lymphoid leukemia was on a 'wait and watch' strategy. In addition, all patients but two (92\%) had additional chronic medical conditions. In particular, 17 (68\%) patients had high blood pressure, $8(32 \%)$ were obese, and $6(25 \%)$ had diabetes mellitus. Fourteen (56\%) patients had more than one coexisting condition besides the hematologic disease.

As reported elsewhere [1], lymphopenia was common at hospital admission $(n=23,92 \%)$, with a median lymphocyte count of 760/ $\mathrm{LL}$ (range, 150-5910). Only one patient had severe neutropenia at the time of COVID-19 diagnosis (median, 2,350/ $\mathrm{LL}$; range, 70-11,400). A computerized tomographic scan of the chest was performed in 14 patients and bilateral ground glass opacities were evident in all of them. A chest radiography was performed in seven additional patients and all radiographs showed bilateral pulmonary opacities.
As of April 16, 2020, with a median follow-up since symptom onset of 29 days (range, 14-40), 13 of the 18 patients $(52 \%)$ developed acute respiratory distress syndrome (ARDS) [9] and 6 received mechanical ventilation (Supplementary Fig. 1). It was decided not to transfer the remaining seven patients with ARDS to the intensive care unit because of their age and hematological disease history. All patients who did not develop ARDS were alive at last follow-up. Of patients with ARDS, nine died, including two who received mechanical ventilation. The Kaplan-Meier estimate of overall survival at 1 month was $60 \%$.

It is hypothesized that similarly to patients with solid malignancies, those with hematologic neoplasms are more susceptible to COVID-19 and develop severe forms. This study highlighted the following observations: patients with a hematologic malignancy harbored a higher risk of developing a severe form of COVID-19 with ARDS, requiring mechanical ventilation, compared to those in the general French population without an underlying medical condition [1]. This translated into a very high mortality (estimated as $40 \%$ at 1 month) which we can expect to be even higher with a longer follow-up. Furthermore, fewer than half of the patients were receiving active anti-neoplastic treatment before COVID19 , highlighting that vigilance must remain high in every patient given the long-term immunosuppressive effect of prior therapies. Interestingly, for the majority of the patients, a nosocomial origin was suspected, owing to their hospitalized status or to outpatient visits within the 14 previous days.

We observed an overrepresentation of patients with MM in our cohort (although MM is not overrepresented in our department), suggesting that such patients are particularly vulnerable, owning to the immunosuppression associated with the disease and its treatment, in particular steroids. In fact, the detrimental effect of steroids on patient outcome has been established during previous coronavirus outbreaks (SARS-CoV-1 and MERS-CoV) [10, 11], and a similar impact is expected in patients infected with SARS-CoV-2 [12]. Finally, we must emphasize that more than half of the patients were over 65 years of age, and $92 \%$ had at least one additional comorbidity, factors which have been associated with COVID-19 severity [1,2], and which have possibly contributed to the seriousness of the infection and high mortality rate observed in our study.

Overall, patients with hematologic malignancies appear to be a population very vulnerable to COVID-19 infection. Therefore, hematology departments should remain COVID19 free zones dedicated solely to hematologic treatment. Furthermore, patients should strictly comply with social distancing and hospital outpatient visits should be reduced to mitigate the risk of COVID-19. 


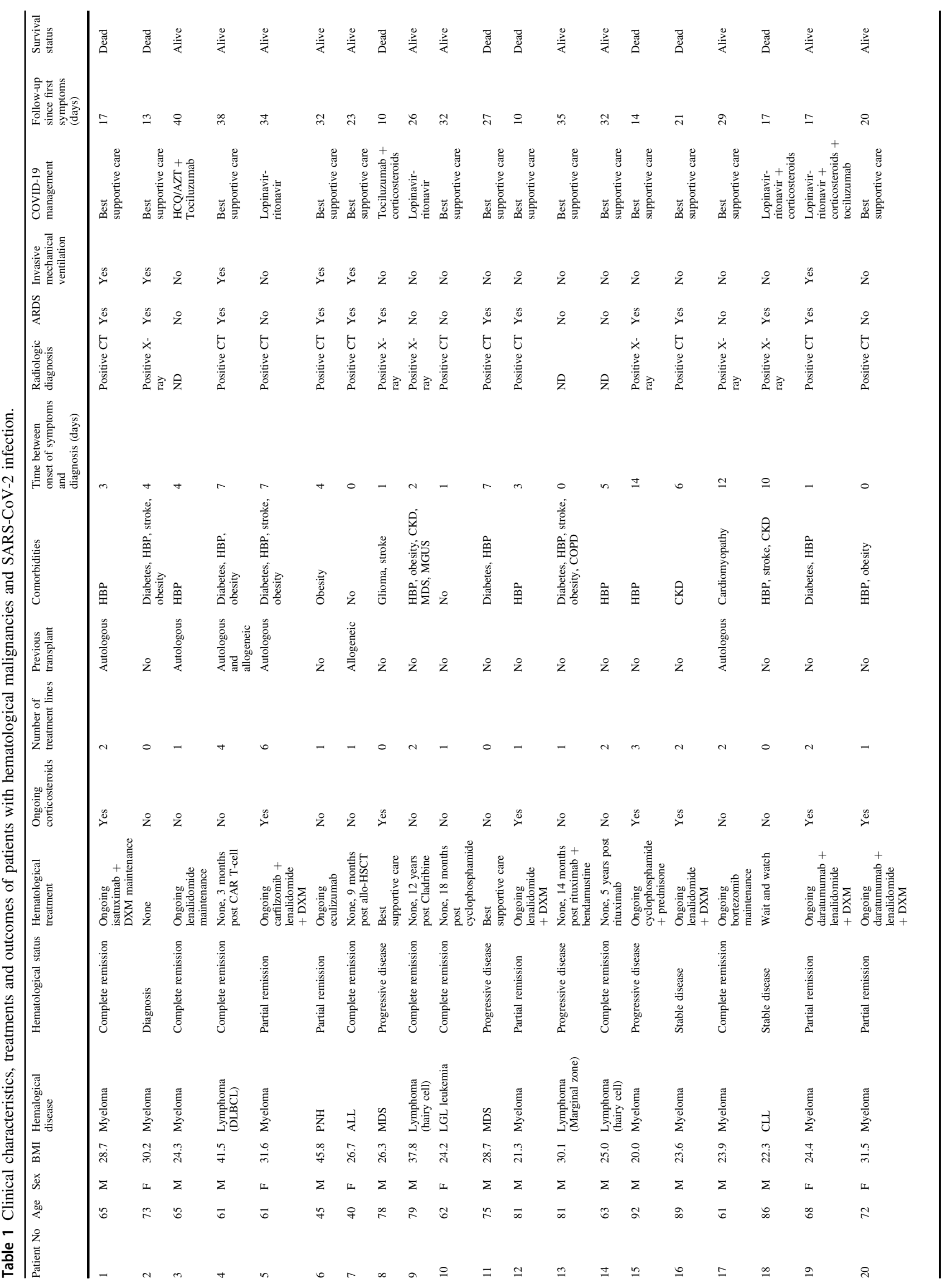




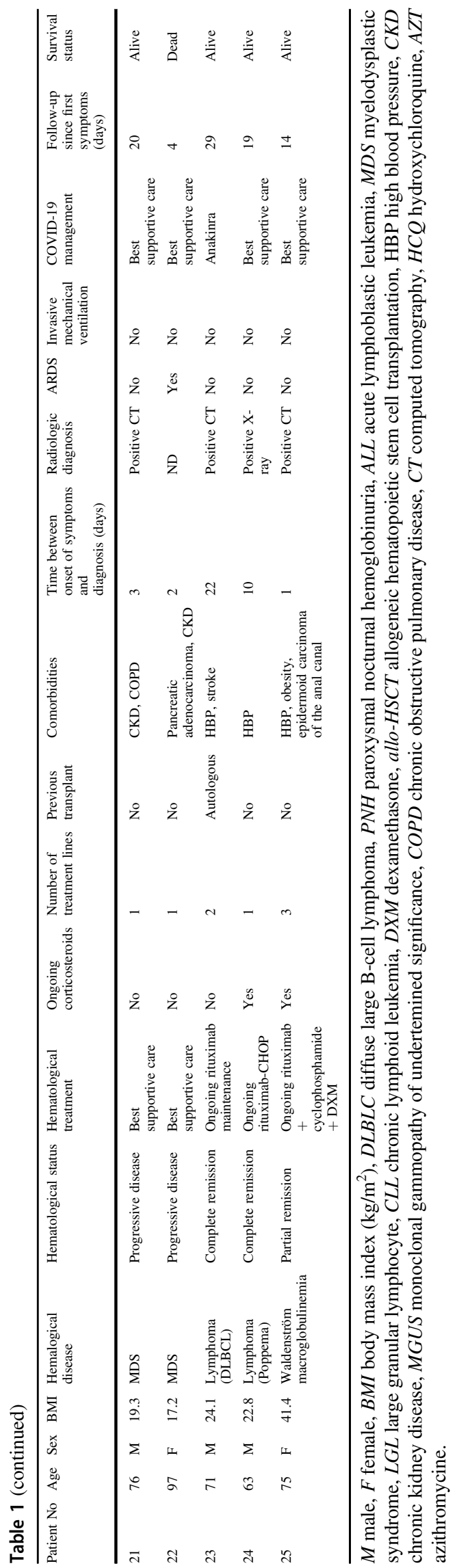

Acknowledgements The authors acknowledge the Association for Training, Education, and Research in Hematology, Immunology, and Transplantation (ATERHIT) for the generous and continuous support of our research work. We thank Dr Tounes Ledraa, Anne Vekhoff, Paul Coppo, Marie-Paule Lemonnier, and our nursing staff for offering the best care to our patients. We thank Prof. Junia V. Melo for critical reading of the manuscript.

Author contributions FM designed the study, recruited patients, collected, assembled, and analyzed the data, and wrote the manuscript. AG recruited patients, collected, assembled, and analyzed the data and helped writing the manuscript. EB, ZW, ZM, SI, AB, SL, SS, EC, AP, $\mathrm{RA}, \mathrm{FMB}$, and OL recruited patients, collected data, and approved the manuscript. ML performed statistical analysis and helped writing the manuscript. RD and MM designed the study, recruited patients, assembled, and analyzed the data, supervised research, and wrote the manuscript.

\section{Compliance with ethical standards}

Conflict of interest MM reports grants and/or lecture honoraria from Janssen, Sanofi, Maat Pharma, JAZZ pharmaceutical, Celgene, Amgen, BMS, Takeda, Pfizer, and Roche, all outside the submitted work. FM reports lecture honoraria from Therakos/Mallinckrodt, Biocodex, Janssen, Keocyt, Sanofi, JAZZ pharmaceutical and Astellas, all outside the submitted work. RD reports lecture honoraria from Keocyt, Sanofi, and Novartis, all outside the submitted work. The other authors declare no competing financial interests.

Publisher's note Springer Nature remains neutral with regard to jurisdictional claims in published maps and institutional affiliations.

\section{References}

1. Guan WJ, Ni ZY, Hu Y, Liang WH, Ou CQ, He JX, et al. Clinical characteristics of coronavirus disease 2019 in China. N Engl J Med. 2020;382:1708-20.

2. Yang X, Yu Y, Xu J, Shu H, Xia J, Liu H, et al. Clinical course and outcomes of critically ill patients with SARS-CoV-2 pneumonia in Wuhan, China: a single-centered, retrospective, observational study. Lancet Respir Med. 2020. In press.

3. Lauer SA, Grantz KH, Bi Q, Jones FK, Zheng Q, Meredith HR, et al. The incubation period of coronavirus disease 2019 (COVID19) from publicly reported confirmed cases: estimation and application. Ann Intern Med. 2020. In press.

4. Koo JR, Cook AR, Park M, Sun Y, Sun H, Lim JT, et al. Interventions to mitigate early spread of SARS-CoV-2 in Singapore: a modelling study. Lancet Infect Dis. 2020. In press.

5. Liang W, Guan W, Chen R, Wang W, Li J, Xu K, et al. Cancer patients in SARS-CoV-2 infection: a nationwide analysis in China. Lancet Oncol. 2020;21:335-7.

6. Yu J, Ouyang W, Chua MLK, Xie C. SARS-CoV-2 transmission in patients with cancer at a tertiary care hospital in Wuhan, China. JAMA Oncol. 2020. In press.

7. Jin XH, Zheng KI, Pan KH, Xie YP, Zheng MH. COVID-19 in a patient with chronic lymphocytic leukaemia. Lancet Haematol. 2020;7:e351-2.

8. Zhang X, Song K, Tong F, Fei M, Guo H, Lu Z, et al. First case of COVID-19 in a patient with multiple myeloma successfully treated with tocilizumab. Blood Adv. 2020;4:1307-10.

9. Ranieri VM, Rubenfeld GD, Thompson BT, Ferguson ND, Caldwell E, Fan E, et al. Acute respiratory distress syndrome: the Berlin definition. JAMA. 2012;307:2526-33. 
10. Lee N, Allen Chan KC, Hui DS, Ng EK, Wu A, Chiu RW, et al. Effects of early corticosteroid treatment on plasma SARSassociated Coronavirus RNA concentrations in adult patients. J Clin Virol. 2004;31:304-9.

11. Arabi YM, Mandourah Y, Al-Hameed F, Sindi AA, Almekhlafi GA, Hussein MA, et al. Corticosteroid therapy for critically ill patients with middle east respiratory syndrome. Am J Respir. Crit Care Med. 2018;197:757-67.

12. Russell CD, Millar JE, Baillie JK. Clinical evidence does not support corticosteroid treatment for 2019-nCoV lung injury. Lancet. 2020;395:473-5. 